\title{
Project MIRACLE: Increasing empathy among psychosocial support staff working with refugees through brief training in motivational interviewing
}

\author{
Miriam Potocky ${ }^{1}$ \& Kristen L. Guskovict ${ }^{2}$ \\ ${ }^{1} \mathrm{PhD}$, School of Social Work, Florida International University, Miami, Florida, USA, ${ }^{2} \mathrm{MS}$, MSW, Heart of Aid Work, Miami, Florida, USA
}

\section{Abstract}

Motivational interviewing (MI) is presented as a mental health and psychosocial support (MHPSS) approach for increasing empathy among psychosocial support staff working with refugees in resettlement. In a pilot study, 34 case managers in US refugee resettlement non-governmental organizations (NGOs) were trained in MI in a 3-h webinar format using a randomized controlled trial with a wait-list condition. Outcome was measured using the Helpful Responses to Refugees Questionnaire, which assesses empathetic responses to common refugee scenarios. Training group participants' responses significantly improved from before to after training compared to the wait-list group which received no training; these results were subsequently replicated in the wait-list group after those participants received training. Pre-post effect sizes were medium to large. Participants reported that the training was useful and relevant, and that they applied the skills in their practice. Barriers and facilitators to use were reported. This pilot study had several limitations, including that the implementation of empathetic responses, their impact on the quality of the case manager-refugee relationship, and the ultimate impact on refugee outcomes could not be assessed. Implications for practice, NGO policy, future research and global MHPSS refugee programmes are discussed.

Keywords: Empathy, MHPSS, motivational interviewing, Project MIRACLE, refugees

\section{KEY IMPLICATIONS FOR PRACTICE}

- Brief MI training has demonstrated promise for enhancing empathy among psychosocial staff working with refugees

- MI should be more broadly considered in MHPSS work in both emergency and non-emergency refugee settings

- A webinar format is a cost-effective training approach that may be particularly relevant in conflict-affected areas globally.

\section{INTRODUCTION}

The global population of forcibly displaced persons, including 23 million refugees, reached a record high in 2016 (United Nations, 2017). Since 1975, over 3 million refugees from more than 70 countries have been resettled in the United States. During the 5-year period from 2013 to 2017, 55,000 to 85,000 refugees were resettled in the United States annually. The five major countries of origin of these refugees were all conflict-affected areas: Iraq, Burma, the Democratic Republic of the Congo, Somalia and Bhutan (Refugee Processing Center, 2017). Despite recent bans and cutbacks on refugee admissions by the federal executive branch, the United States continues to maintain a comprehensive and coordinated programme for resettlement assistance, administered through the State

\begin{tabular}{|l|l|}
\hline \multicolumn{2}{|c|}{ Access this article online } \\
\hline Quick Response Code: & Website: \\
& www.interventionjournal.org \\
& \\
&
\end{tabular}

Department's Bureau of Population, Refugees, and Migration and the Department of Health and Human Services' Office of Refugee Resettlement. This programme funds case management services to assist refugees with the multiplicity of challenges they face in their new lives, including employment, education, language, acculturation, health and mental health. The resettlement programme aims to help refugees 'maximize their potential in the United States by linking them to critical resources that

Address for correspondence: Dr. Miriam Potocky, School of Social Work, Florida International University, Modesto Maidique Campus, AHC5-569, Miami, FL 33199, USA.

E-mail: potockym@fiu.edu

This is an open access journal, and articles are distributed under the terms of the Creative Commons Attribution-NonCommercial-ShareAlike 4.0 License, which allows others to remix, tweak, and build upon the work non-commercially, as long as appropriate credit is given and the new creations are licensed under the identical terms.

For reprints contact: reprints@medknow.com

How to cite this article: Potocky M., \& Guskovict. K.L. (2019). Project MIRACLE: Increasing empathy among psychosocial support staff working with refugees through brief training in motivational interviewing. Intervention, 17(1), 59-68. 
Potocky and Guskovict: Project MIRACLE

assist them in becoming integrated members of American society'(U.S. Office of Refugee Resettlement, 2018).

\section{The role of case managers}

The federal resettlement programme funds the delivery of refugee case management services through non-governmental organizations (NGOs). Case managers focus on helping clients meet their basic needs and adapt to their new lives. Incoming refugees experience multiple stressors, including acculturation compounded with the traumas of war and migration, and decision-making regarding available services and life choices such as employment.

Case managers provide psychosocial support in the context of the first and second tiers of the four-layer intervention pyramid devised by the United Nations' Inter-Agency Standing Committee (IASC) on mental health and psychosocial support (MHPSS) in emergency settings [InterAgency Standing Committee (IASC), 2007]. The first, foundational, layer comprises basic services and security, and the second addresses community and family supports. Case managers' function is to link clients to these necessary resources. Although the IASC MHPSS guidelines were developed for emergency situations, a critical review of the application of MHPSS with refugees has noted that MHPSS must also be addressed in non-emergency, urban settings such as those common in resettlement (Meyer, 2013).

Empathy has been identified as an important, but often missing, element of humanitarian assistance for refugees (Morand, 2013). Our prior research has substantiated this, finding that case managers represent diverse backgrounds (including some former refugees themselves) and diverse levels of training and professional affiliation. Although many possess undergraduate or graduate degrees, only a minority of these degrees are in helping professions such as psychology, counselling or social work, despite these workers' role as psychosocial support providers. Those workers without a degree in a helping profession demonstrated less empathy than their counterparts. Moreover, empathy was not correlated with length of employment either with refugees specifically or in human services in general (Potocky \& Guskovict, 2016).

All of these findings suggest a need for training in empathy skills. This paper presents a pilot study on training resettlement case managers in the use of motivational interviewing (MI) to enhance their empathetic skills in working with refugees. Although there are other techniques besides MI that can be used to enhance empathy, MI was selected for this context, because it extends beyond empathy into empowering clients to maximize their potential, which is the ultimate aim of refugee resettlement, as identified above.

This pilot study is an initial phase of a planned longer term endeavour, Project MIRACLE: Motivational Interviewing for Refugee Adaptation, Coping, and Life Empowerment. A logic model illustrating the current and planned phases of Project MIRACLE, as well its assumptions and relevant external factors, is shown in Figure 1. MI is detailed below.

\section{Motivational interviewing}

MI (Miller \& Rollnick, 2013) is a client-centred, humanistic approach designed to engage reluctant clients who are ambivalent about change. Its core tenets, or 'spirit', are collaboration, evocation, autonomy and compassion. The four practice principles of MI are expressing empathy, developing discrepancy between client goals and behaviours, rolling with resistance (i.e. avoiding confrontation), and supporting client self-efficacy.

Although MI was originally developed in the field of addiction treatment, it has since spread to many other areas of health, MHPSS (Arkowitz, Miller, \& Rollnick, 2015; Hohman, 2011; Rollnick, Miller, \& Butler, 2008) and has been identified as a promising practice for refugee resettlement (Potocky, 2016). The conceptual framework for the application of MI with refugees rests on several rationales. First, similar to persons with addictions, although refugees may see the necessity for change, they are sometimes reluctant to adapt, given that they have not freely chosen to do so, but have been forced into it. Abdullah Mohammad, a recently resettled Syrian refugee, exemplified this perspective:

He was not an immigrant who set out to adapt to a new world; he was a refugee trying to hold on to what had been ripped from him. 'We're forced to be here', Mr. Mohammad said. 'We're happy, but we're forced to be here.' (Einhorn \& Kantor, 2016, para 22).

Second, refugees and their case managers sometimes disagree on the goals of their work plan, due to differing worldviews and expectations. As stated in a joint report issued by the United Nations High Commissioner for Refugees, the International Organization for Migration and MHPSS.net, 'helpers may focus solely on what they think needs to be done, without sufficient attention to how their activities may be experienced by refugees and migrants' (Ventevogel, Shinina, Strang, Gagliato, \& Hansen, 2015, p. 4). For example, well-intentioned case managers often try to persuade clients to seek counselling for their trauma; however, for many refugees, counselling or psychiatric care are unfamiliar, stigmatized or tools of political oppression - so naturally, these clients refuse. The case manager and client then engage in a tug-of-war. MI is specifically designed to avoid such a battle of wills.

Third, MI focuses on building trust between the caseworker and client. This is vital for refugees who will naturally be distrustful of authority figures because of their past experiences.

Research shows that the existence of trust, which emerges from empathy within a working alliance, is a critical predictor of successful outcome in a helping relationship (Laska, Gurman, \& Wampold, 2014; Miller \& Rollnick, 2013; Miller \& Rollnick, 2013). Fourth, MI has been utilized worldwide, demonstrating its cross-cultural relevance and hence its potential applicability to refugees from around the globe. Of particular interest is view of the fact that most contemporary refugees in the United States are racial or ethnic minorities; a meta-analysis of 72 MI studies spanning a range of 


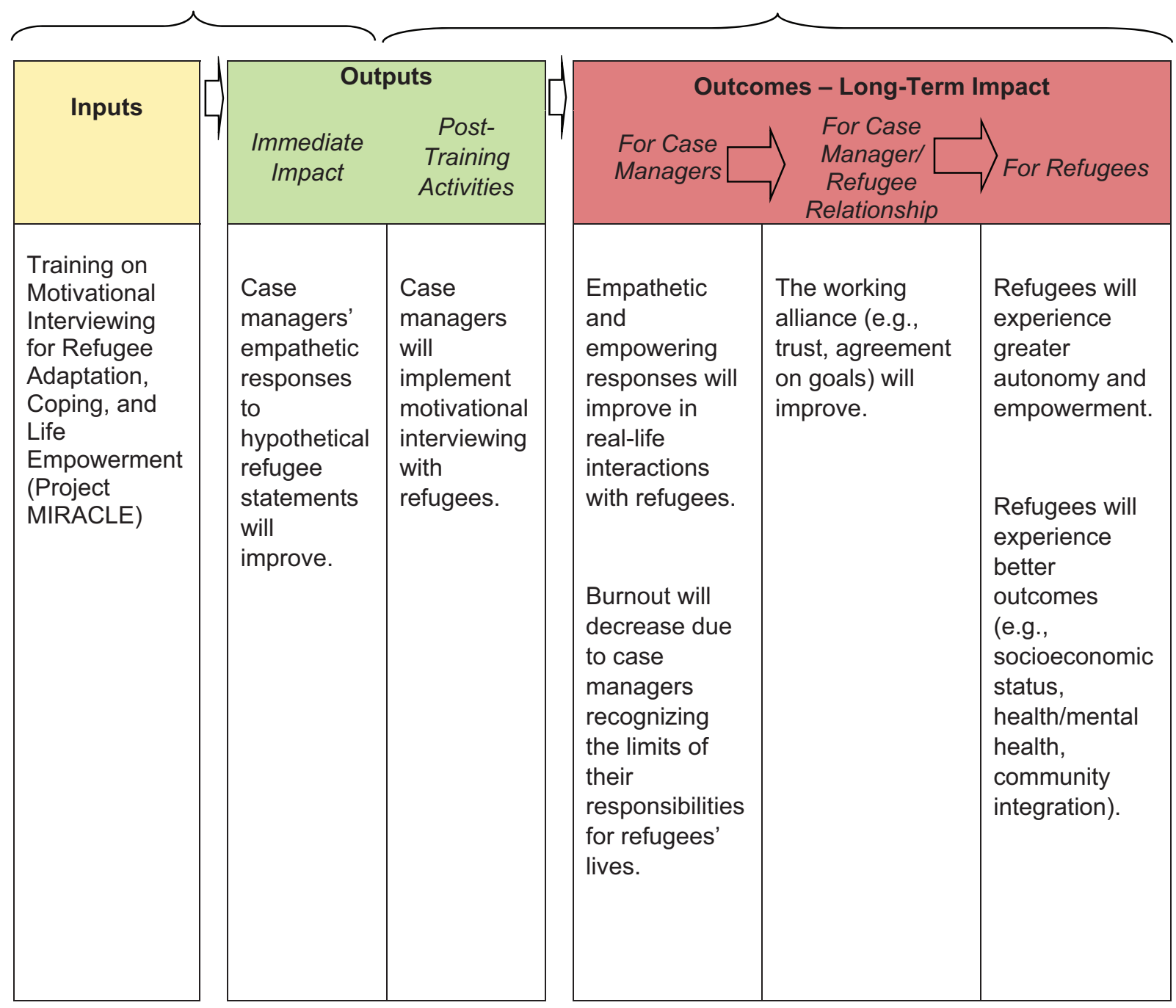

Assumptions
Refugees experience ambivalence about
change.
Case managers on average have low skills in
motivational interviewing techniques; such
skills can be learned and implemented.

Figure 1: Project MIRACLE logic model

target problems found effect sizes almost twice as large among minority populations compared to non-minority populations (Hettema, Steele, \& Miller, 2005).

Fifth, because of its stress on client autonomy, MI frees caseworkers from taking too much responsibility and doing everything for their clients and instead empowers clients to make their own decisions. Respect for autonomy has been identified as a key principle for working with refugees (Ventevogel et al., 2015). Although some decisions, such as returning to one's native country, are out of refugees' control, many others, such as financial, employment, educational, child-rearing and other life skill decisions, are ones that they must learn to navigate in their new lives. Finally, approaches such as the MI

\section{External Factors}

The evolving socio-political-cultural-organisational environment will influence both case managers and refugees.

Screening, Brief Intervention, and Referral to Treatment (Substance Abuse and Mental Health Services Administration, 2017) have shown that MI can be used in brief interactions, which makes it ideal for resettlement case managers to incorporate into their work. Resettlement workers often have only 2 or 3 months to assist their clients and carry high caseloads, further limiting their available time. They do not provide therapy; instead, they provide a feeling of safety through the psychosocial support and linkage to resources to meeting basic needs.

Empathy is a core component of MI and as noted earlier, it is critical for working with refugees. The developers of MI (Miller \& Rollnick, 2013) have speculated that the 
reason MI may be more effective with persons from minority backgrounds is because the experience of having a compassionate listener in a formal helping role may be more unusual for them. However, there are significant barriers to empathy, including over-identification and burn-out, both of which speak directly to the resettlement case management experience. If a resettlement worker has had a similar experience to an incoming refugee, the worker may not have a great deal of empathy for the way in which the newly arrived refugee is managing his or her own experience. And case managers' high caseloads are associated with burnout, which decreases empathy.

\section{XXMI training}

MI has been established as an evidence-based practice by the Cochrane and Campbell Collaborations and National Registry of Evidence-Based Programmes and Practices of the US Substance Abuse and Mental Health Service Administration, and training on MI has proliferated. Several systematic reviews and meta-analyses of MI training studies agree that MI training produces medium-to-large short-term effect size impacts on practitioner behaviours as assessed by a variety of outcome measurements (Barwick, Bennett, Johnson, McGowan, \& Moore, 2012; de Roten et al., 2013; Madson, Loignon, \& Lane, 2009; Schwalbe, Oh, \& Zweben, 2015). Training typically is provided in didactic and interactive workshops with a wide range of exposure from 2 to $40 \mathrm{~h}$. Significantly, only one previous study was identified in which training was provided in a distance learning format (Shafer, Rhode, \& Chong, 2004), as was the case in the current study, and no previous study has reported on MI training for psychosocial support staff working with refugees.

\section{Study purpose}

The purpose of this pilot study was to determine the effect of a brief MI training webinar on participants' empathetic responses to hypothetical refugee statements. Participants were randomly assigned to training and wait-list groups. The training group completed an outcome questionnaire before and after the training (Times 1 and 2); the wait-list group completed the instrument at the same time points. The wait-list group subsequently completed the training and then completed the questionnaire once again after the training (Time 3 ). It was hypothesized that

(1) The training group's mean score will improve more from Time 1 to 2 than the waitlist group's mean score.

(2) The wait-list group's change in mean score from Time 2 to 3 will show a comparable improvement to that of the training group from Time 1 to 2 .

\section{Materials AND MEthodS \\ Ethics}

This study was approved by the Institutional Review Board of the authors' institution.

\section{Research design and sample size}

This study was a randomized controlled trial with training and wait-list conditions.

Power analysis indicated that with a medium effect size $(f)$ of $0.25, \alpha$ of 0.05 and power of 0.80 , a total sample size of 34 was required to test the primary hypothesis (Hypothesis 1) (Faul, Erdfelder, Lang, \& Buchner, 2007). To compensate for potential drop-outs, the study aimed to enrol approximately 20 participants each in the training and wait-list groups, for a total sample size of 40 .

\section{Sample}

The target participants were individuals who worked directly with refugees in the resettlement process. In early 2016, an invitation to the webinar was sent to a list of 435 email addresses of personnel in NGOs serving refugees throughout the United States, harvested from the website of the US Office of Refugee Resettlement and links therein. The e-mail provided a brief overview of the webinar, noted who the target participants were, noted that completion of pre- and post-tests was required, and noted that participation was limited. To enrol, interested participants clicked a link that took them to an online pre-test. The pre-test first contained two screening questions: (1) Do you work directly with refugees? (Yes/no); and if yes, (2) What is your role within your work with refugees? (Direct service/ administrative).

Respondents who answered either 'no' to question (1) or 'administrative' to question (2) were disqualified. Qualified participants proceeded to the rest of the questionnaire (see 'Instrumentation' section below) and upon completion were randomized to either the training or wait-list group. The webinar was scheduled to take place approximately 1 month following the e-mail invitation.

The participant flow data are shown in Figure 2. As seen, 119 persons initially enrolled and were randomized (61 to the intervention group and 58 to the wait-list group). Nineteen participants allocated to the training group received the training, and 17 of those completed the post-test. Wait-list participants were asked to complete the same questionnaire again at the time that the webinar started for the training group participants, and $25 \mathrm{did}$ so; of these, eight had missing or invalid data (described below under 'Instrumentation' section), resulting in 17 for analysis. Consequently, data from 34 participants (17 each in the training and wait-list groups) were ultimately analysed for Hypothesis 1; thus, the required sample size was attained.

Wait-list participants were offered the webinar following their completion of their second questionnaire; 15 of the 17 wait-list participants received the training and 10 of those completed the post-test, and their data were analysed for Hypothesis 2. Upon completion of all pre- and post-tests, participants were provided a Certificate of Completion.

Finally, approximately one month following the webinar, all 34 participants who had received the training, regardless of whether they completed the post-test (i.e. 19 in the original training group and 15 in the wait-list group) were 
Potocky and Guskovict: Project MIRACLE

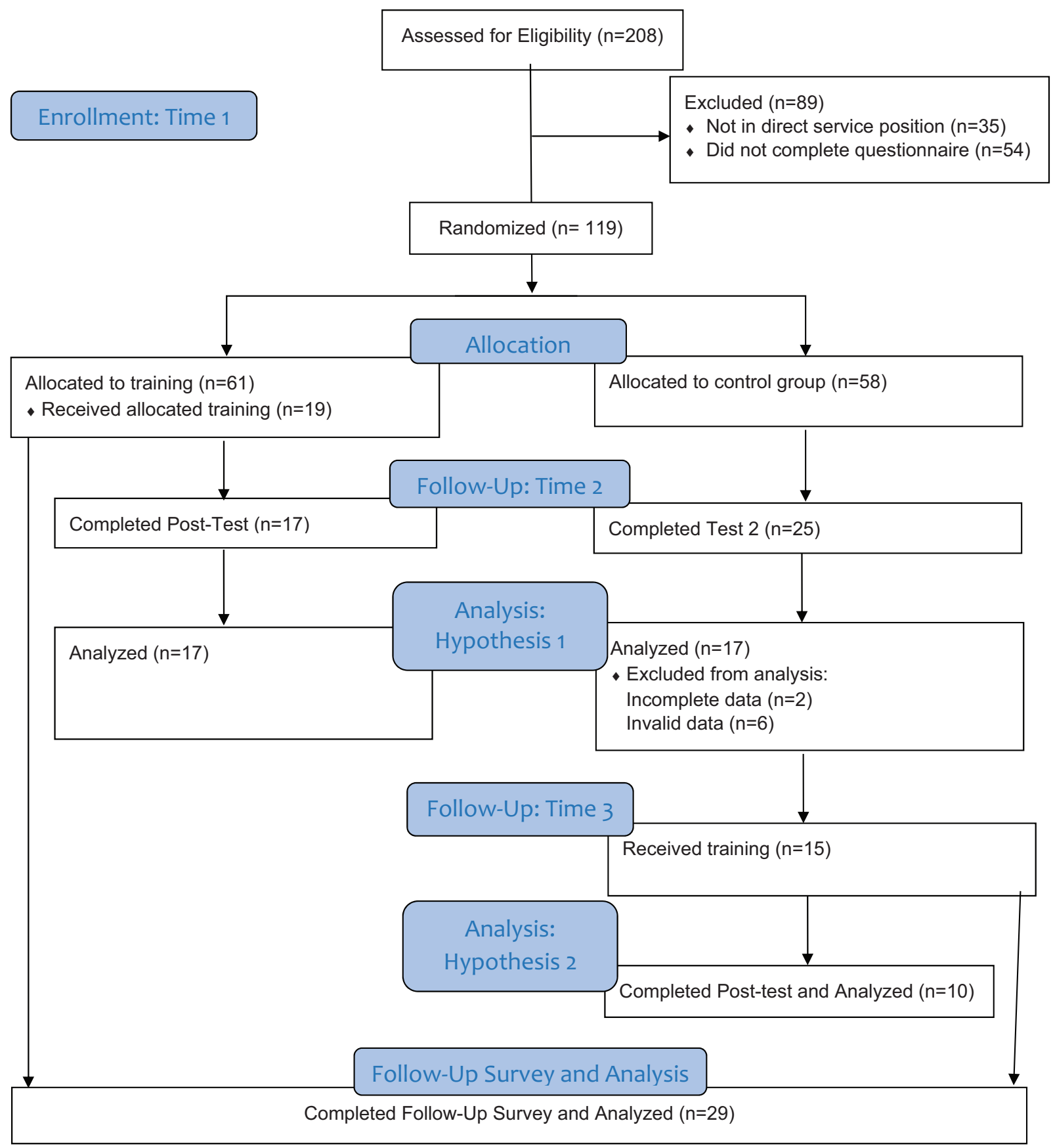

Figure 2: Participant flow diagram

asked to complete a follow-up survey; 29 did so and their data were analysed. Following completion of this survey, these respondents received a $\$ 10$ Starbucks electronic gift card in appreciation of their time.

\section{Training}

The training provided in this study consisted of two 90-min webinar sessions delivered 1 week apart. The topics covered the background, rationale and basic principles and skills of MI, as shown in Table 1. Powerpoint slides combined with extensive oral presentation by the two authors were used. The authors grounded the abstract concepts with specific examples relevant to refugee clients. These examples were drawn from the authors' extensive practice, scholarly and personal experiences (the first author is a former refugee). In addition, several brief video clips were used that were obtained from YouTube, to provide variety in the learning experience. These videos were not specific to refugee clients but were specific to the skills or concepts at hand (e.g. empathy, active listening).

Further, a role play was enacted during the first session to demonstrate empathetic response skills. Subsequently, participants were provided with a scenario and asked to provide their own empathetic responses. In between the two sessions, participants were paired and instructed to consult with each other during the week about situations in their practice, wherein they were able to use the skills learned in session 1. They were then asked to share these experiences at the start of the second session. Finally, throughout each session, participants were provided several opportunities to ask questions and provide examples. 
Potocky and Guskovict: Project MIRACLE

\begin{tabular}{ll}
\hline Table 1: Motivational interviewing (MI) webinar training content & \\
\hline Part I & Part II \\
\hline What MI is and is not & Review of concepts from Part I \\
Evidence base for MI & Active listening \\
Rationale for MI with refugees & Open questions, affirmations \\
Spirit and principles of MI & Reflections, summaries (OARS) \\
Importance of empathy and how to show it & Developing discrepancy \\
Empathy in action - rolling with resistance role play and participant exercise & MI in action with refugees - participant exercise
\end{tabular}

\section{Table 2: Helpful Responses to Refugees Questionnaire}

The following five paragraphs are things that a refugee might say to you. With each paragraph, imagine that a refugee you know is talking to you and explaining a problem that he or she is having. You want to help by saying the right thing. Think about each paragraph. For each paragraph, write the next thing you might say if you wanted to be helpful. Write only one or two sentences for each situation

1. A 43-year-old resettled male refugee says

'I can only find part-time low-paying jobs. Because I cannot speak English, no one wants to hire me. My wife doesn't mind working cleaning hotels, but there is no respect in that for a man. My children have to come with me to translate everywhere I go. You know how hard it is to learn English at my age? And how can I support my family? I sometimes feel so angry . . . no, I don't want to talk about it . . .

2. A 30-year-old female refugee says

'I can't sleep at night - I keep having nightmares of how my brother was dragged out of our home and executed in front of us by the military. And I miss my big family back home - here, I have no one. I feel very lonely. Counseling? No, that's for crazy people'

3. A 15-year-old refugee girl says

'I only went to school twice last week because my mother was sick and I had to go with her to the doctor's, so I could help the doctor understand what was wrong. The week before, my mom got some work, and so I had to stay home from school to watch my little brother while she worked. I've missed so much school I don't think I will pass my classes. It might be best if I just drop out and get a job so I can help my parents pay the bills

4. A 22-year-old refugee woman says

'My son is 3 years old, and sometimes he does not listen to anything I tell him. I used to hit him with a wire hanger when he wouldn't listen. That's how my mom raised me. But, a woman in the neighborhood told me that my child could be taken from me if I do that again. I just laughed - he is my son, and when he disobeys it is my responsibility to teach him the right way to behave'

5. A 38-year-old female refugee says

'My husband was arrested for hitting me and I don't understand why. He didn't kill me, so I don't know why he's being punished. Now with him in jail, I'm having a really hard time caring for our four children. They refuse to obey me. And now we don't have my husband's income. And I don't drive or speak English - my husband took care of those things. I need my husband back'

\section{Instrumentation}

All questionnaires were completed online using Qualtrics Software (Qualtrics, Seattle, WA, USA). The pre- and post-test measure was the Helpful Responses to Refugees Questionnaire (HRRQ), a previously validated instrument designed to measure empathetic responsiveness among refugee service providers via simulation (Potocky \& Guskovict, 2016). The HRRQ is a refugee-specific adaptation of the Helpful Responses Questionnaire used by MI researchers, which is a proxy measure of empathy that has been demonstrated to correlate with actual MI skills in real-world application (Miller, Hedrick, \& Orlofsky, 1991; Miller \& Mount, 2001). The HRRQ [Table 2] consists of five scenarios that are reflective of statements that refugee clients might typically make. Respondents are instructed to write a one-to-two sentence open-ended response to each scenario indicating what they would say to the imaginary client. Responses are then rated on a fivepoint scale from least to most helpful (i.e. empathetic). Total scores thus range from 5 to 25 .

At pre-test, participants were also asked to provide data on their level and field of education. Data on length of work experience was not gathered because this was not found to be correlated with empathy in the previous study described earlier.
The two authors coded all responses to the HRRQ. All responses were disarticulated and randomized so that coders were blind to which respondent an item came from, group condition and time of administration (Times 1, 2 or 3). This was performed to eliminate bias in coding, because the authors conducted both the training and the coding. Upon reading the responses, it was discovered that within the wait-list group, two respondents had not answered all questions, and six had not followed the questionnaire instructions to 'write the next thing you might say if you wanted to be helpful'. These six respondents wrote what they would do rather than what they would say (e.g. 'I will call police') or provided a diagnostic assessment (e.g. 'anxious, depressed'). Thus, these six were considered invalid responses. These eight cases were excluded from subsequent analysis [Figure 1].

In addition, there were four instances in which respondents stated what they would do rather than say in response to one of the five questions (they stated what they would say in response to the other four). In these instances, rather than exclude the entire case, the mean of the four other items was substituted for the one anomalous item.

Inter-coder reliability, as assessed by intra-class correlation for all items across all respondents and all time points, was 0.82. Coding discrepancies were resolved by discussion, 
Potocky and Guskovict: Project MIRACLE

while still blind, and a final code was assigned. After final coding, internal consistency, as assessed by the Cronbach's $\alpha$ for the pre-test scores, was 0.83 .

The follow-up survey administered approximately 1 month following the webinar consisted of four closed-ended questions about the usefulness and relevance of the webinar, with five Likert scale response options. Further, one question asked whether the respondent had applied the skills used in the webinar, with a yes or no response option. Finally, two open-ended questions asked about barriers encountered in applying the skills and factors that enhanced use of the skills.

\section{Data analysis}

All quantitative data were analysed using SPSS software (International Business Machines Corp., Armonk, NY, USA). To test Hypothesis 1, a repeated measures analysis of variance was conducted to examine the time $\times$ group interaction between the training and wait-list groups on the HRRQ mean scores at Times 1 and 2.

To test Hypothesis 2, paired-samples $t$ tests were conducted on the HRRQ scores at Times 1 and 2 for the training group and Times 2 and 3 for the wait-list group. In addition, Cohen's $d$ effect sizes for these within-group mean differences were computed.

For the follow-up questionnaire, descriptive statistics were computed for the closed-ended questions. The open-ended questions were qualitatively analysed using content analysis to identify the most frequently occurring themes.

\section{RESULTS}

\section{Sample characteristics}

The sample characteristics for the 34 participants who ultimately were entered into analysis for Hypothesis 1 are shown in Table 3. There were no significant differences between the training and wait-list groups on either education level or field.

\section{Hypothesis 1}

At Time 1, the HRRQ mean scores of the training group (M $=11.9, \mathrm{SD}=5.5)$ and wait-list group $(\mathrm{M}=12.2, \mathrm{SD}=6.3)$ were nearly identical. However, at Time 2, the mean score of the training group had increased to 17.8 ( $\mathrm{SD}=6.7$ ), whereas the mean score of the wait-list group remained virtually unchanged $(\mathrm{M}=12.8, \mathrm{SD}=5.6)$. This was a significant group $\times$ time interaction effect, $F(1,32)=11.7$, $P=0.002$, thereby supporting Hypothesis 1 .

\section{Hypothesis 2}

The above-described increase in HRRQ mean scores from Time 1 to 2 for the training group was significant, $t(16)=$ $4.7, P=0.004$; this was a large effect size, $d=1.1$. Among the 10 respondents in the wait-list group who subsequently completed the webinar and the HRRQ at Time 3, mean scores increased from $12.9(\mathrm{SD}=5.8)$ at Time 2 to 16.5 $(\mathrm{SD}=7.4)$ at Time 3 . This was also significant, $t(9)=2.8$,

\begin{tabular}{lc}
\hline Table 3: Sample characteristics $(\boldsymbol{N}=\mathbf{3 4})$ & \\
\hline Characteristic & $\boldsymbol{n}(\%)$ \\
\hline Educational level & \\
Some college & $1(2.9)$ \\
Associate's degree & $1(2.9)$ \\
Bachelor's degree & $18(52.9)$ \\
Master's degree & $10(29.4)$ \\
Doctorate degree & $2(5.9)$ \\
Unknown & $2(5.9)$ \\
Educational field & \\
Social work & $7(20.6)$ \\
Education & $4(11.8)$ \\
International/regional studies & $4(11.8)$ \\
Science/engineering & $4(11.8)$ \\
Health & $4(11.8)$ \\
Other/unknown & $4(11.8)$ \\
Counselling/psychology & $3(8.8)$ \\
Social science & $3(8.8)$ \\
No degree & $1(2.9)$ \\
\hline
\end{tabular}

$P=0.022$, with a medium effect size, $d=0.6$. Although the wait-list group attained a smaller mean score after training than the training group, this difference was not statistically significant, $t(25)=0.5, P=0.651$. Therefore, Hypothesis 2 was supported.

\section{Follow-up}

Table 4 shows the responses to the quantitative follow-up questions. As seen, the vast majority of the 29 participants who responded to the follow-up survey agreed or strongly agreed that the webinar was relevant to their work with refugees, was useful in dealing with their challenges in their work, enhanced their skills, was useful to their career and enabled them to serve their clients better. The vast majority also reported having applied what they learned in the webinar in their work.

\section{Barriers}

Participants were asked to list any barriers they had encountered when performing MI with refugees. The five most frequently listed barriers were language/interpretation, low client educational levels, client unwillingness to engage or to change, lack of time and culture.

Examples of statements made in relation to each of these themes were

\section{Language/interpretation}

(1) Translation of some of the jargon into terms that are rich in meaning in the clients' languages.

(2) Our largest barrier is language. Most do not have the English skills necessary to communicate as well as needed, that is emotional language, motivational language, goals etc.

(3) Difficulties with interpreters understanding what we're trying to do. Sometimes I am not sure carefully chosen words are interpreted as accurately as possible. 
Potocky and Guskovict: Project MIRACLE

\begin{tabular}{lc}
\hline Table 4: Follow-up responses $(\boldsymbol{N}=\mathbf{2 9})$ & \\
\hline Statement & $\boldsymbol{n}(\%)$ \\
\hline The webinar was relevant to my work with & \\
refugees & \\
Strongly agree & $16(55.2)$ \\
Agree & $12(41.4)$ \\
Neutral & $1(3.5)$ \\
Disagree & $0(0)$ \\
Strongly disagree & $0(0)$ \\
The material presented in the webinar has been & \\
useful to me in dealing with the challenges I face & \\
working with refugees & \\
Strongly agree & $8(27.6)$ \\
Agree & $19(65.5)$ \\
Neutral & $1(3.5)$ \\
Disagree & $1(3.5)$ \\
Strongly disagree & $0(0)$ \\
The webinar enhanced my skills in this topic area & \\
Strongly agree & $15(51.7)$ \\
Agree & $13(44.8)$ \\
Neutral & $0(0)$ \\
Disagree & $1(3.5)$ \\
Strongly disagree & $0(0)$ \\
This webinar was relevant to my career & $18(62.1)$ \\
Strongly agree & $10(34.5)$ \\
Agree & $1(3.5)$ \\
Neutral & $0(0)$ \\
Disagree & $0(0)$ \\
Strongly disagree & \\
The webinar has enabled me to serve my clients & \\
better & \\
Strongly agree & $14(4.5)$ \\
Agree & $12(41.4)$ \\
Neutral & $2(6.9)$ \\
Disagree & $1(3.5)$ \\
Strongly disagree & \\
Have you applied any of what you learned in the \\
webinar to your work? \\
Yes & \\
No & \\
\hline & \\
& \\
\hline
\end{tabular}

(4) At times, it is difficult to use MI with interpreters. Some open-ended questions may require abstract thinking and the interpreter may ask the question in a more concrete fashion (i.e. leaving less room for elaboration). It is a slower process towards the goal of the session; a lot of rephrasing has to be performed.

\section{Low client educational levels}

(1) Many refugees have not had formal education, so questions that require a detailed response are very difficult for them to answer. I continue to receive no response to open-ended questions, which forces me to turn them into one- or two-word-answer questions.

\section{Client unwillingness to engage or change}

(1) When a client is unwilling to engage with me.

(2) One barrier I have encountered during MI is when a client is not at a point of self-determination and is unable to identify clear goals.
(3) There have been barriers of non-interest in the client to change their situation. This created the challenge of then rolling with resistance to figure out which direction the client wanted to go in.

(4) My refugee students are reluctant to open up (for a variety of reasons).

(5) Lack of time.

(6) I don't always see a client for a long time to go through the whole sequence.

(7) Building rapport with client needs time.

(8) Culture.

(9) Cultural differences.

(10) Culture.

\section{Facilitators}

Respondents were also asked to list any factors which had enhanced their use of MI with refugees. The five most frequently listed facilitating factors were empathy, using reflections/active listening, rolling with resistance, sharing MI techniques with others and patience/taking time. These are reflected in the comments below:

\section{Empathy}

(1) Continued use of empathy and understanding that a refugee did not leave her/his country by choice and has to deal with consequential changes.

(2) The focus on empathy really resonated with me. I think I have been sympathetic and sometimes empathetic in speaking with refugees, but I tend to try to cheer people up and point out the bright side of difficult situation. I realized through this training that the way.

(3) I can be most helpful to refugees who are struggling and try to understand their struggle and empathize, rather than minimize it.

(4) The way I communicate with my client is better now that what it was when I began. I am able to listen to my client and be able to put myself in that client's shoes. Before it was hard for me to put myself in the client's perspective. Using MI has been really helpful. Also it works for the client because he feels that he is being heard.

\section{Using reflections/active listening}

(1) Trying to validate what a refugee is saying and giving them time to explain their situation, even if I don't have immediate answers or help, seems to be the best approach.

(2) They react best if they feel they are being listened to and understood.

(3) I have been able to respond more effectively to refugees making what I would perceive as poor decisions. Rather than reacting right away, I take more time (if I have time) to ask clarifying questions and reflect feelings back to the client.

(4) Taking note of body language, remembering to reiterate and/or summarize what clients have said to let them know we're listening. 


\section{Rolling with resistance}

(1) I have been reminded that I cannot take ownership of my client's problem. I cannot offer my advice as 'normal' either. I really appreciated being reminded of how important perspective is when refugees encounter difficult situations in the United States.

(2) I think that realizing that resistance was part of the process has enhanced my use of MI.

(3) Sharing MI techniques with others.

(4) Sharing the information about MI with our staff.

(5) Discussions with the other staff that deal with refugees and sharing ideas and experiences.

(6) Patience/taking time.

(7) It helps me to take a step back and remain patient.

(8) Allowing more time for my intake process. Spending some time getting to know the refugees as I do the intake.

\section{Discussion}

This study has demonstrated that a brief MI training webinar produces an immediate, short-term improvement in refugee resettlement workers' empathetic skills as measured by a proxy instrument. The present study is consistent with prior studies showing that MI training produces medium-to-large short-term effects on practitioner skills. This is the first time that MI training has been applied and tested specifically for psychosocial support staff working with this population. Further, the webinar approach used in this study supports the efficacy of distance education, a cost-effective staff training option; this may be particularly relevant for conflict-affected areas, which are globally dispersed in low- and middle-income countries.

Respondents' feedback to the follow-up questions about barriers and facilitators to the use of MI in their work yielded some intriguing findings. One of these was the reported challenges posed by linguistic differences and interpretation issues. One respondent mentioned translation of jargon; it seems that clarification during training may be needed, as jargon should not be a part of MI application. The reported challenges regarding interpreters suggest that interpreters should also receive the MI training, so that they are prepared for what to expect in an MI session and why, allowing them to interpret accurately.

In regard to factors that facilitated the use of MI, respondents primarily noted MI principles or skills themselves - empathy, reflections/active listening and rolling with resistance.

These responses indicate that participants were able to apply these skills in relation to refugees, thus moving from abstract concepts to concrete manifestations. The external facilitating factors mentioned - discussing the techniques with others and taking time - indicate that NGOs should make these resources available for their staff to support use of MI with their refugee clients.

\section{Limitations}

There are a number of limitations to this pilot study. First, this study assessed only empathy skills and no other MI skills such as developing discrepancy. It is possible that training in helping approaches other than MI may have likewise improved empathy scores. Similarly, respondents' comments about client unwillingness to engage or to change appear to reflect a basic disconnect about the foundational ideas of MI and suggest that greater development in additional MI skills or more time for their deployment (as noted by other respondents) is needed.

Further, respondents' comments about culture as a barrier are perplexing as respondents did not provide any elaboration about this, and in light of the fact, noted earlier, that MI has successfully been used in many cultures around the world. Thus, this topic requires further exploration to identify exactly what the perceived cultural barriers are and to potentially adapt the MI training programme accordingly. Moreover, the validity of the follow-up questionnaire is unknown; the responses may have been intended to please the researchers.A methodological issue also requiring further exploration is the response rate. Out of 119 individuals initially randomized, 34 received the training and completed the post-test without missing or invalid data. This seemingly low completion rate is likely attributable to the fact that the webinar training took place 4 to 6 weeks after enrolment and randomization (depending on group assignment). Although it was initially thought that this would allow participants to set aside time in their busy schedules for the training, it is possible that those same busy and unpredictable schedules interfered with enrollees' intentions to participate. Further, the lack of a registration fee (even a token one) or supervisory encouragement to participate may have created unintended disincentives to participation despite the initial interest. Ultimately, it is not known how those who followed through with participation differed from those who did not.

Further limitations of this research arise from the limited resources available for this pilot study. One of these limitations is that the empathy skills could not be assessed in vivo; rather, they were assessed using a proxy measure. Future research should assess real-world practitioner behaviours with clients using intervention fidelity monitoring tools such as the MI Treatment Integrity Coding Manual, which entails audio- or video-recording practitioner/client interactions and coding them for utilization of MI skills (Moyers, Manuel, \& Ernst, 2015). In addition, research is needed to determine whether the application of MI skills in fact improves the relationship between case managers and refugees. This can be measured using tools such as the Working Alliance Inventory (Horvath, 1992). Another resource-related limitation is that participants' skills could not be assessed in the longer term. Previous research shows that post-workshop training is needed to maintain skills gained (Schwalbe, Oh, \& Zweben, 2015). Finally, this pilot study did not allow for the examination of the ultimate impact of the training on client outcomes, such as well-being and social integration. All of these are areas for future research within the larger planned scope of Project MIRACLE.

\section{ConcLusion}

This pilot study of brief training in MI for psychosocial support staff working with resettled refugees in the United 
States has demonstrated promising results suggesting that the use of MI should be more broadly considered and investigated in MHPSS contexts involving refugees in emergency as well as non-emergency settings around the world.

\section{Presentation at a meeting}

Nil.

\section{Financial support and sponsorship}

This study was supported by New York Community Trust.

\section{Conflicts of interest}

There are no conflicts of interest.

\section{REFERENCES}

Arkowitz, H., Miller, W. R., \& Rollnick, S. (Eds.) (2015). Motivational interviewing in the treatment of psychological problems (2nd ed.). New York, NY: Guilford.

Barwick, M. A., Bennett, L. M., Johnson, S. N., McGowan, J., \& Moore, J. E. (2012). Training health and mental health professionals in motivational interviewing: A systematic review. Children and Youth Services Review, 34, 1786-1795.

de Roten, Y., Zimmerman, G., Ortega, D., \& Despland, J. (2013). Metaanalysis of the effects of MI training on clinicians' behavior. Journal of Substance Abuse Treatment, 45, 155-162.

Einhorn, C., \& Kantor, J. (2016, December 17). Wonder and worry, as a Syrian child transforms. The New York Times. Retrieved from: https://www.nytimes.com/2016/12/17/world/americas/syrian-refugees-canada.html.

Faul, F., Erdfelder, E., Lang, A-G., \& Buchner, A. (2007). G*Power 3: A flexible statistical power analysis program for the social, behavioral, and biomedical sciences. Behavior Research Methods, 39, 175-191.

Hettema, J., Steele, J., \& Miller, W. R. (2005). Motivational interviewing. Annual Review of Clinical Psychology, 1, 91-111.

M. Hohman (Ed.) (2011). Motivational interviewing in social work practice. New York, NY: Guilford.

Horvath, A. O. (1992). Working alliance inventory. Retrieved from: http:// wai.profhorvath.com/.

Inter-Agency Standing Committee (IASC) (2007). IASC guidelines on mental health and psychosocial support in emergency settings. Geneva: IASC. Retrieved from https://interagencystandingcommittee.org/system/files/ legacy_files/Guidelines\%20IASC\%20Mental\%20Health\%20Psychosocial $\% 20 \% 28$ with $\% 20$ index $\% 29$.pdf

Laska, K. M., Gurman, A. S., \& Wampold, B. E. (2014). Expanding the lens of evidence based practice in psychotherapy: A common factors perspective. Psychotherapy, 51, 467-481.
Madson, M. B., Loignon, A. C., \& Lane, C. (2009). Training in motivational interviewing: A systematic review. Journal of Substance Abuse Treatment, 36, 101-109.

Meyer, S. (2013). UNHCR's mental health and psychosocial support. Geneva: UNHCR. Retrieved from http://www.unhcr.org/51bec3359. pdf

Miller, W. R., \& Mount, K. A. (2001). A small study of training in motivational interviewing: Does one workshop change clinician and client behavior? Behavioural and Cognitive Psychotherapy, 29, 457-471.

Miller, W. R., \& Rollnick, S. (2013). Motivational interviewing: Helping people change (3rd ed.). New York, NY: Guilford.

Miller, W. R., Hedrick, K. E., \& Orlofsky, D. R. (1991). The helpful responses questionnaire: A procedure for measuring therapeutic empathy. Journal of Clinical Psychology, 47, 444-448.

Morand, M. (2013). Acknowledgement. In S. Meyer, (Ed.), UNHCR's mental health and psychosocial support. Geneva: UNHCR. Retrieved from http://www.unhcr.org/51bec3359.pdf

Moyers, T. B., Manuel, J. K., \& Ernst, D. (2015). Motivational interviewing treatment integrity coding manual 4.2.1. Retrieved from http://motivationalinterviewing.org/sites/default/files/miti4_2.pdf

Potocky, M. (2016). Motivational interviewing: A promising practice for refugee resettlement. Journal of Ethnic and Cultural Diversity in Social Work, 25, 247-252.

Potocky, M., \& Guskovict, K. (2016). Enhancing empathy among humanitarian workers through Project MIRACLE: Development and initial validation of the Helpful Responses to Refugees Questionnaire. Torture Journal, 26, 46-59.

Refugee Processing Center (2017). Refugee admissions report November 30, 2017. Retrieved from http://www.wrapsnet.org/admissions-andarrivals/

Rollnick, S., Miller, W. R., \& Butler, C. (2008). Motivational interviewing in health care. New York, NY: Guilford.

Schwalbe, C. S., Oh, H. Y., \& Zweben, A. (2015). Sustaining motivational interviewing: A meta-analysis of training studies. Addiction, 109, 1287-1294.

Shafer, M. S., Rhode, R., \& Chong, J. (2004). Using distance education to promote the transfer of motivational interviewing skills among behavioral health professionals. Journal of Substance Abuse Treatment, 26, 141-148.

Substance Abuse and Mental Health Services Administration (2017). Screening, brief intervention, and referral to treatment. Retrieved from https://www.samhsa.gov/sbirt

U.S. Office of Refugee Resettlement (2018). Homepage. Retrieved from http://www.acf.hhs.gov/orr

United Nations (2017). UNHCR figures at a glance. Retrieved from http:// www.unhcr.org/en-us/figures-at-a-glance.html

Ventevogel, P., Shinina, G., Strang, A., Gagliato, M., \& Hansen, L. J. (2015). Mental health and psychosocial support for refugees, asylum seekers, and migrants on the move in Europe. Geneva: International Organization for Migration. Retrieved from https://www.iom.int/sites/default/files/our_work/DMM/MigrationHealth/MP_infosheets/MHPSS-refugees-asylum-seekers-migrantsEurope-Multi-Agency-guidance-note.pdf 